\title{
Weed Management in Secondary Nursery of \\ Tectona grandis and Pterocarpus santalinus
}

\author{
Shahbaz Noori* and Ramesh Rathod \\ Department of Silviculture and Agroforestry, College of Forestry Sirsi (University of \\ Agricultural Sciences, Dharwad) 581401, India
}

*Corresponding author

Keywords

Pre-emergence,

Post-emergence,

Secondary nursery,

Weed control,

Weed

population, Diuron,

Pendimethalin.

\section{Article Info}

Accepted:

18 August 2019

Available Online:

10 September 2019

\section{A B S T R A C T}

Testing techniques to reduce weed infestation is a crucial step in developing direct tree seedling systems. The use of pre-emergence herbicides may be an alternative to manual weeding techniques, but so far, information on how they affect native tree species based nurseries is scarce. We established an experiment to know the major weed flora and evaluated the effect of pre and post emergent herbicide on weed suppression of secondary nursery of Tectona grandis and Pterocarpus santalinus. A total of 17 weed species belonging to 12 families were recorded among which Synedrella nudiflora, Bidens pilosa, Cynodon dactylon and Mallow species were more frequently recorded. Overall our results suggest that, of all tested herbicides reduced weed cover. Of the tested herbicide, Diuron and Pendimethalin showed the most effective weed control indicating that future experiments should increase the number of species tested as well as investigate how seed traits can affect the species response to different herbicides.

\section{Introduction}

The out-planting of seedlings in tree nurseries is the principal method for establishing woodlands in India where the use of natural regeneration is not viable. This is partly because the problems of seed predation and weed competition are more easily addressed in an intensively managed nursery site (Willoughby et al., 2004a). In nursery production, weeds can compete with tree seedlings for light, moisture and nutrients, which can kill small, recently emerged seedlings.

Hand weeding is costly in nurseries although costly, but is not a practical option on extensive direct seeded sites (Willoughby, 1996). Thus, use of herbicides is an attractive and cost effective option for many managers. 
A wide variety of tree species are raised at forest nurseries for afforestation programmes. In southern India, Tectona grandis (Teak) and Pterocarpus santalinus (Red Sander) are widely grown as block and boundary plantation. Teak and Red Sander are valued for its durability and water resistant, decay resistant and medicinal properties that is attractive to wood based industry and farmers. In order to meet heavy requirement of seedlings supply to wood based industries, farmers, for meeting needs of social forestry and afforestation programmes, production of healthy seedlings is a prerequisite. Most forest restoration techniques involve nursery raised seedlings, especially in tropical areas; however, this option is often too costly to be used in large area (Rodrigues et al., 2011).

Weed competition usually reduced seedling establishment and growth (Pereira et al., 2013). This is especially problematic in the first year after sowing, when seedlings have very small root systems (Willoughby et al., 2003). Moreover, weeds often build up a viable soil seed bank, making initial management of restored sites very difficult and challenging (D' Antonio and Meyerson, 2002). Using of pre-emergence prior to sowing of tree species and post emergence herbicide could be a feasible option to improve seeding system. It can reduce or eliminate weed seed bank and therefore decrease competition by weeds (Jinks et al., 2006). Most commercial brands of preemergence herbicides are not selective for grasses, but affect a wide range of herbaceous plants (Andrei, 2013).

Therefore it is essential to identify effective pre and post emergence herbicide which are not harmful to nursery seedlings used in restoration. Although pre-emergence herbicide effects have been tested in a few studies in temperate areas (Willoughby et al., 2006), there is no information about how they effectively suppress weeds during seedling emergence and early growth of forest nursery seedlings. This lack of information inhibits the use of pre-emergence herbicides in forest nurseries of tropical areas.

The present study was investigated the major weed flora and the effect of pre-emergence and post emergence herbicides on Teak and Red Sander nursery seedlings.

Moreover, we tested the effectiveness of the same herbicides in providing satisfactory weed control by reducing or eliminating weed present in nursery. The aim of the study was to assess the major weed flora and find the most suitable herbicide for effective weed control without compromising on seedling emergence and growth of forest nursery seedlings.

\section{Materials and Methods}

Our experiment was conducted in the forest nursery of College of Forestry, Sirsi (UAS Dharwad), Karnataka, in 2018-19. We adopted complete randomized design (CRD) with 14 treatments and three replicates. Two native species selected were viz., Tectona grandis and Pterocarpus santalinus based on seedling availability and silvicultural characteristics, representing sample seedlings of regional tropical forest nursery. The treatment consisted of four pre-emergent herbicide, combination of four similar pre-emergent with one post emergent herbicide, combinations of pre-emergent herbicides with hand weeding and a control, which was not treated.

Herbicides were selected from the range of formulations recommended for common weeds in the study area and were applied following the recommendations of the manufacturers (Table 1). The 14 treatments selected were: $\mathrm{T}_{1}$ : Pendimethalin 30\% EC @ 3litre/ha, T2: Alachlor 50\% EC @ 2.5 litre/ha, $\mathrm{T}_{3}$ : Butachlor 50\% EC @ 2.5 litre/ha, $\mathrm{T}_{4}$ : 
Diuron 80\%WP @ $1.25 \mathrm{~kg} / \mathrm{ha}, \mathrm{T}_{5}$ : Pendimethalin 30\% EC @ 3 litre/ha + Quizalfop Ethyl 5 EC @ $1000 \mathrm{ml} / \mathrm{ha}$ at 30 DAT (Days after Treatment), $\mathrm{T}_{6}$ : Alachlor 50\% EC @ 2.5 litre/ha + Quizalfop Ethyl 5 EC @ 1000ml/ha at 30 DAT, T: Butachlor 50\% EC @ 2.5 litre/ha + Quizalfop Ethyl 5 EC @ 1000ml/ha at 30 DAT, T: Diuron 80\% WP @ $1.25 \mathrm{~kg} / \mathrm{ha}+$ Quizalfop Ethyl 5 EC @ $1000 \mathrm{ml} / \mathrm{ha}$ at 30 DAT, T9: Pendimethalin $30 \%$ $\mathrm{EC} @ 3$ litre/ha + HW at 30 DAT, T 10 : Alachlor 50\% EC @ 2.5 litre/ha + HW at 30 DAT, $\mathrm{T}_{11}$ : Butachlor 50\% EC @ 2.5 litre/ha + HW at 30 DAT, T 12 : Diuron 80\% WP @ 1.25 $\mathrm{kg} / \mathrm{ha}+\mathrm{HW}$ at 30 DAP, $\mathrm{T}_{13}$ : Once hand weeding at 30 DAT and $\mathrm{T}_{14}$ : Control (No weed control). Before spraying, re-bagging of polybags was done and filled with proper proportion of soil, sand and FYM (1:2:1) and was irrigated for two days.

The data was collected and presented in weed species taxonomy table (Table 2). Six seedlings per treatment were treated using herbicide and data on growth of seedlings and the phyto-toxic effect on weeds were monitored for period of 30 and 60 days of treatment. Weed count, weed dry weight was pooled and weed control efficiency was calculated, subjecting to Fisher's method of ANOCA at 5 per cent significance level to compare the means of treatment and data was interpreted as given by Panse and Sukhatme (1967).

\section{Results and Discussion}

\section{Weed species taxonomy}

Generally, the weed vegetation of an area is determined not only by the environment but also by the edaphic and biological factors that include soil structure, $\mathrm{pH}$, nutrients, moisture status, associated crops, weed control measures and field history especially in local geographic variation (Hakim et al., 2010). A total of 17 different weed species which identified comprising of 13 broadleaved (dicot) and 4 grass (monocot) species in secondary forest nursery of teak and red sander. The perennial broadleaved species were more prevalent than grass species. The weed species represented 6 families in secondary teak nursery with highest in Asteraceae (3) followed by Poaceae (2) Amarantheceae (2). The rest of the families were represented by one species each in secondary nursery of teak. Similarly, in red sander nursery, 10 families of weed species were represented with highest in family Poaceae (3) followed by Asteraceae (2) and Amaranthaceae (2). The rest families were represented by one species. Synedrella nudiflora, Bidens pilosa, Cynodon dactylon and Mallow species were more frequently recorded weed flora in secondary nursery (Table 2).

\section{Weed count and Weed dry weight}

Weed management treatments significantly influenced weed count and its dry weight at 60 DAT. All weed control treatments were found significantly superior to un-weeded control $\left(\mathrm{T}_{14}\right)$. In secondary nursery of Red Sander, minimum numbers of weeds were observed at 15 DAT, 30 DAT, 45 DAT and 60 DAT were in treatment of $\mathrm{T}_{8}$ (0.92/poly bag), $\mathrm{T}_{7}$ (1.25/poly bag), $\mathrm{T}_{12}$ (1.25/poly bag) and $\mathrm{T}_{12}$ (1.57/poly bag) respectively. Maximum weeds numbers were recorded in un-weeded check (18.87/poly bag) at 60 DAT.

Similarly, in secondary nursery of teak, the minimum number was observed at 15 DAT in treatment of $\mathrm{T}_{7}(0.42 /$ poly bag), and at 30 DAT, 45 DAT and 60 DAT were recorded in treatment of $\mathrm{T}_{9}(0.75 /$ poly bag), (0.6/poly bag) and (1.2/poly bag) respectively. Maximum weeds number were recorded in un-weeded check (11.08/poly bag), than hand weeding at 60 DAT (5.17/poly bag) (Table. 3 ).

Weed dry weight at 60DAT, un-weeded check recorded significantly higher dry weight 
(0.82g/poly bag) compared to all other treatments which could be due to higher uninterrupted growth while lowest was observed in $\mathrm{T}_{12}$ treatment $(0.163 \mathrm{~g} /$ poly bag $)$ in secondary nursery of Red sander. Similarly in secondary nursery of teak, at 60 DAT, unweeded check $\left(T_{14}\right)$ recorded significantly higher dry weight of weeds $(1.175 \mathrm{~g} /$ poly bag) than rest of the treatments. Significantly lower dry weight of weeds was observed in $T_{9}$ $(0.25 \mathrm{~g} /$ poly bag) treatment (Table. 4$)$.

\section{Weed control efficiency (\%)}

Data on weed control efficiency revealed that, at 60 DAT, highest weed control efficiency $(80.08 \%)$ was recorded in treatment $\mathrm{T}_{12}$ in secondary nursery of Red sander (Diuron as the primary spray and hand weeded at 30 DAT), followed by $\mathrm{T}_{9}(78.65 \%)$ treatment. The lower weed control efficiency $(42.68 \%)$ was recorded in treatment that received Butachlor only as a primary spray $\left(\mathrm{T}_{3}\right)$.

In secondary nursery of teak, at 60 DAT, in secondary nursery of teak, highest weed control efficiency $(78.72 \%)$ was recorded in treatment $\mathrm{T}_{9}$ (Pendimethaline as the primary spray and hand weeded at $30 \mathrm{DAT}$ ), followed by $\mathrm{T}_{11}(69.15 \%)$ treatment. However, lower weed control efficiency (41.27\%) was recorded in treatment that received only Alachlor $\left(T_{2}\right)$ as a pre-emergent spray.

The higher weed control efficiency could be attributed to the lower weed count and lower dry weight in all the recorded treatments.

\section{Effect of herbicides on seedling height and collar diameter}

Data on seedling height revealed that, in secondary nursery of red sander, at 2 MAT (months after treatment), maximum percent increment in height $(15.04 \%)$ was recorded in $\mathrm{T}_{12}$ (Diuron 80\%WP @ $1.25 \mathrm{~kg} / \mathrm{ha}+\mathrm{HW}$ at 30 DAP) with plant height of $19.9 \mathrm{~cm}$, followed by Treatment $\mathrm{T}_{6}(15.03 \%)$, which follows the application of Alachlor 50\% EC @ 2.5 litre/ha + Quizalfop Ethyl 5 @ 1000ml/ha as a post -emergent herbicide. Further treatments $\mathrm{T}_{9}$ and $\mathrm{T}_{11}$ showed same increment $(14 \%)$ in the height of respective treated seedling and least increment in seedling height was recorded in control $(6.7 \%)$. In secondary nursery of teak, at 2 MAT, maximum percent increment in seedling height $(21.05 \%)$ was recorded in $\mathrm{T}_{9}$ (Pendimethalin 30\% EC @ 3litre/ha + hand weeding at 30 DAP) attaining height of $26.33 \mathrm{~cm}$, followed by treatment $\mathrm{T}_{12}$ (14.14\%), which follows the application of Diuron $80 \%$ WP @ $1.25 \mathrm{~kg} / \mathrm{ha}+\mathrm{HW}$ at 30 DAP. The least increment in seedling height was recorded in control (3.54\%). The maximum seedling height attained by teak and red sander may be due to reduced weed population, weed dry weight and higher weed control efficiency as recorded in above treatments.

Data on collar diameter revealed that in secondary nursery of red sander at 2 MAT, maximum percent increment of collar diameter $(32.9 \%)$ was recorded in $\mathrm{T}_{12}$ (Diuron $80 \%$ WP @ $1.25 \mathrm{~kg} / \mathrm{ha}+\mathrm{HW}$ at $30 \mathrm{DAP})$ attaining diameter of $0.598 \mathrm{~cm}$, followed by Treatment $\mathrm{T}_{6}$ (32.16\%), which follows the application of Alachlor 50\% EC @ 2.5 litre/ha +Quizalfop Ethyl 5@ 1000ml/ha at 30 DAT.

The treatments $\mathrm{T}_{1}(16 \%)$ and $\mathrm{T}_{3}(16.5 \%)$ shows lower increment percents and least increment in collar diameter was recorded in control condition (7.5\%). Similarly, in secondary nursery of teak, maximum percent increment of collar diameter at 2 MAT $(25.4 \%)$ was recorded in $\mathrm{T}_{9}$ (Pendimethalin 30\% EC @ 3litre/ha + hand weeding at 30 DAP) attaining a collar diameter of $0.627 \mathrm{~cm}$, followed by Treatment $\mathrm{T}_{5}(20.21 \%)$. The treatments $\mathrm{T}_{7}(7.39 \%)$ and $\mathrm{T}_{8}(8.57 \%)$ shows lower increment percents and least increment in collar diameter was recorded in control $(5 \%)$. 
Table.1 Pre emergence and Post emergence herbicide details

\begin{tabular}{|c|c|c|c|}
\hline $\begin{array}{c}\text { Active } \\
\text { ingredient }\end{array}$ & $\begin{array}{c}\text { Commercial } \\
\text { product }\end{array}$ & Mechanism of action & $\begin{array}{c}\text { Application } \\
\text { rate }\end{array}$ \\
\hline Pendimethalin & Pendimyd & $\begin{array}{c}\text { Prevents cell division and elongation in } \\
\text { susceptible species }\end{array}$ & 3 litre/ha \\
\hline Alachlor & Lasso & $\begin{array}{c}\text { Effect on root growth and plant protein } \\
\text { production }\end{array}$ & 2.5 litre/ha \\
\hline Butachlor & Rasayan chlor & Effect on root development & 2.5 litre/ha \\
\hline Diuron & Diuron 50\% WP & Effect on photosynthesis & $1.25 \mathrm{~kg} / \mathrm{ha}$ \\
\hline Quizalfop Ethyl & Targa super & Effect on grass growth & $1000 \mathrm{ml} / \mathrm{ha}$ \\
\hline
\end{tabular}

Table.2 Weed species taxonomy in secondary nursery of Teak and Red Sander

\begin{tabular}{|l|c|c|c|c|}
\hline \multicolumn{5}{|c|}{ Weed species taxonomy in secondary nursery of Teak } \\
\hline Family & Common name & Scientific name & Life cycle & Morphology \\
\hline Poaceae & Bidari hullu & Oplismenus burmani & A & Grassy \\
\hline Poaceae & Bermuda grass & Cynodon dactylon & A & Grassy \\
\hline Commelinaceae & Day flower & Commelina diffusa & A & Grassy \\
\hline Asteraceae & Synedrella & Synedrella nudiflora & P & Broad leaved \\
\hline Amarantheceae & Dwarf copper weed & Alternanthera sessilis & P & Broad leaved \\
\hline Cucurbitaceae & Chitrati & Mukia maderaspsta & P & Broad leaved \\
\hline Asteraceae & Goat weed & Ageratus conyzoids $L$ & P & Broad leaved \\
\hline Amarantheceae & Pigweed & Amaranthus viridis & P & Broad leaved \\
\hline Asteraceae & Spanish needle & Bidens pilosa & P & Broad leaved \\
\hline Oxalidaceae & Wood sorrel & Oxylis corniculata & P & Broad leaved \\
\hline & Weed species taxonomy in secondary nursery of Red Sander & \\
\hline Poaceae & Bidari hullu & Oplissmenus burmanii & A & Grassy \\
\hline Poaceae & Crab grass & Digitaria ciliaris & A & Grassy \\
\hline Poaceae & Bermuda grass & Cynodon dactylon & A & Grassy \\
\hline Asteraceae & Goat weed & Ageratus conyzoides $L$ & P & Broad leaved \\
\hline Euphorbiaceae & Turike balli & Tragia involucrate & P & Broad leaved \\
\hline Rubiaceae & Button weed & Spermacoce ocymoides & P & Broad leaved \\
\hline Cucurbitaceae & Chitrati & Mukia maderaspata & P & Broad leaved \\
\hline Urticaceae & - & Laportea interupta & P & Broad leaved \\
\hline Lindernaceae & - & Lindernia crustuciae & P & Broad leaved \\
\hline Malvaceae & - & Mallow species & P & Broad leaved \\
\hline Asteraceae & Fire weed & Crassocephalum & P & Broad leaved \\
\hline Oxalidaceae & Wood sorrel & Oxalis corniculata & P & Broad leaved \\
\hline Amaranthaceae & Dwarf copper weed & Altarnanthera sessilis & P & Broad leaved \\
\hline Amaranthaceae & Pig weed & Amaranthus virirdis & P & Broad leaved \\
\hline
\end{tabular}


Table.3 Number of weeds per poly bag at different stages of seedling growth as influenced by weed control treatments in secondary nursery of Red Sander and Teak

\begin{tabular}{|c|c|c|c|c|c|c|c|c|}
\hline \multirow{3}{*}{ Treatments } & \multicolumn{4}{|c|}{ Red Sander } & \multicolumn{4}{|c|}{ Teak } \\
\hline & \multicolumn{4}{|c|}{ Weed count (No. Per poly bag) } & \multicolumn{4}{|c|}{ Weed count (No. Per poly bag) } \\
\hline & 15 DAT & 30 DAT & 45 DAT & 60 DAT & 15 DAT & 30 DAT & 45 DAT & 60 DAT \\
\hline $\mathbf{T}_{1}$ & 1.08 & 1.33 & 1.83 & 2.08 & 1.33 & 2 & 1.9 & 2.3 \\
\hline $\mathbf{T}_{2}$ & 1.08 & 1.88 & 1.93 & 2 & 1.42 & 1.75 & 2.58 & 4.08 \\
\hline $\mathbf{T}_{3}$ & 1.58 & 1.67 & 2 & 4.33 & 0.92 & 0.92 & 1.58 & 2.05 \\
\hline $\mathbf{T}_{4}$ & 1.67 & 3.42 & 3.33 & 3.75 & 2.92 & 3.75 & 4.5 & 4.57 \\
\hline $\mathbf{T}_{5}$ & 1.42 & 2.17 & 2.33 & 2.85 & 1.67 & 1.75 & 1.8 & 2 \\
\hline $\mathbf{T}_{6}$ & 1.42 & 2 & 1.48 & 1.66 & 1 & 3.33 & 3.66 & 3.33 \\
\hline $\mathbf{T}_{7}$ & 1 & 1.25 & 3.67 & 5.83 & 0.42 & 1.25 & 2.25 & 4.25 \\
\hline $\mathbf{T}_{8}$ & 0.92 & 3.67 & 3.92 & 5.58 & 1.75 & 2 & 4.75 & 3.17 \\
\hline $\mathbf{T}_{9}$ & 1.75 & 2.17 & 1.5 & 1.63 & 0.58 & 0.75 & 0.6 & 1.2 \\
\hline $\mathbf{T}_{10}$ & 1.58 & 3.08 & 2.08 & 3.42 & 0.5 & 2.08 & 2.02 & 3.17 \\
\hline $\mathbf{T}_{11}$ & 1.33 & 2.25 & 2.25 & 5.42 & 0.5 & 1.25 & 1.25 & 2.58 \\
\hline $\mathbf{T}_{12}$ & 1.42 & 2.67 & 1.25 & 1.57 & 0.83 & 3.75 & 2.5 & 2.5 \\
\hline $\mathbf{T}_{13}$ & 7.42 & 12.92 & 5.67 & 9.58 & 1.44 & 6.5 & 7.01 & 5.17 \\
\hline $\mathbf{T}_{14}$ & 8.92 & 17.25 & 18.75 & 18.87 & 3.12 & 9.92 & 9.75 & 11.08 \\
\hline S.E.m \pm & 0.51 & 1.07 & 0.74 & 0.16 & 0.40 & 0.64 & 0.42 & 0.45 \\
\hline C.D. at $5 \%$ & NS & 3.32 & 2.29 & 0.35 & 1.23 & 1.96 & 1.31 & 1.40 \\
\hline
\end{tabular}


Table.4 Effect of weed control treatments on weed dry weight at different stages of seedling growth in secondary nursery of Red sander and Teak.

\begin{tabular}{|c|c|c|c|}
\hline \multirow{2}{*}{\multicolumn{2}{|c|}{ Treatments }} & \multicolumn{2}{|c|}{$\begin{array}{l}\text { Weed dry weight } \\
\text { (gram/poly bag) }\end{array}$} \\
\hline & & \multirow{2}{*}{$\begin{array}{c}\text { Red sander } \\
0.333\end{array}$} & \multirow{2}{*}{$\begin{array}{l}\text { Teak } \\
0.51\end{array}$} \\
\hline $\mathbf{T}_{1}$ & Pendimethalin 30\% EC @ 3litre/ha & & \\
\hline $\mathbf{T}_{2}$ & Alachlor 50\% EC @ 2.5 litre/ha & 0.445 & 0.69 \\
\hline $\mathbf{T}_{3}$ & Butachlor 50\% EC @ 2.5 litre/ha & 0.47 & 0.66 \\
\hline $\mathbf{T}_{4}$ & Diuron80\%WP @ 1.25 kg/ha & 0.348 & 0.615 \\
\hline $\mathbf{T}_{5}$ & $\begin{array}{l}\text { Pendimethalin 30\% EC @ } 3 \text { litre/ha +Quizalfop Ethyl } 5 \text { EC@ } \\
\text { 1000ml/ha at } 30 \text { DAP. }\end{array}$ & 0.285 & 0.485 \\
\hline $\mathbf{T}_{6}$ & $\begin{array}{l}\text { Alachlor 50\% EC @ } 2.5 \text { litre/ha + Quizalfop Ethyl } 5 \text { EC@ } \\
\text { 1000ml/ha at } 30 \text { DAP. }\end{array}$ & 0.325 & 0.689 \\
\hline $\mathbf{T}_{7}$ & $\begin{array}{l}\text { Butachlor 50\% EC @ } 2.5 \text { litre/ha + Quizalfop Ethyl } 5 \text { EC@ } \\
\text { 1000ml/ha at } 30 \text { DAP. }\end{array}$ & 0.428 & 0.65 \\
\hline $\mathbf{T}_{8}$ & $\begin{array}{l}\text { Diuron 80\%WP @ } 1.25 \text { kg/ha + Quizalfop Ethyl } 5 \text { EC@ } \\
\text { 1000ml/ha at } 30 \text { DAP. }\end{array}$ & 0.2 & 0.542 \\
\hline $\mathbf{T}_{9}$ & Pendimethalin 30\% EC @ 3litre/ha + HW at 30 DAP. & 0.175 & 0.25 \\
\hline $\mathbf{T}_{10}$ & Alachlor 50\% EC @ 2.5 litre/ha + HW at 30 DAP. & 0.23 & 0.525 \\
\hline $\mathbf{T}_{11}$ & Butachlor 50\% EC @ 2.5 litre/ha + HW at 30 DAP. & 0.227 & 0.408 \\
\hline $\mathbf{T}_{12}$ & Diuron 80\%WP @ 1.25 kg/ha + HW at 30 DAP. & 0.163 & 0.363 \\
\hline $\mathbf{T}_{13}$ & Once hand weeding at $30 \mathrm{DAP}$ & 0.195 & 0.487 \\
\hline $\mathbf{T}_{14}$ & Weedy check (control) & 0.82 & 1.175 \\
\hline & S.E.m $( \pm)$ & 0.063 & 0.091 \\
\hline & C.D. at $5 \%$ level & 0.193 & 0.280 \\
\hline
\end{tabular}

Table.5 Weed control efficiency (\%) in secondary nursery of Red sander and Teak respectively

\begin{tabular}{|c|c|c|c|}
\hline \multicolumn{2}{|r|}{ Treatments } & \multicolumn{2}{|c|}{ Weed Control Efficiency (\%) } \\
\hline & & Red sander & Teak \\
\hline$T_{1}$ & Pendimethalin 30\% EC @ 3litre/ha & 59.35 & 56.59 \\
\hline $\mathbf{T}_{2}$ & Alachlor 50\% EC @ 2.5 litre/ha & 45.73 & 41.27 \\
\hline $\mathbf{T}_{3}$ & Butachlor 50\% EC @ 2.5 litre/ha & 42.68 & 44.04 \\
\hline $\mathbf{T}_{4}$ & Diuron 80\%WP @ 1.25 kg/ha & 57.52 & 47.65 \\
\hline $\mathbf{T}_{5}$ & $\begin{array}{l}\text { Pendimethalin 30\% EC @ } 3 \text { litre/ha +Quizalfop Ethyl } 5 \text { EC@ } \\
\text { 1000ml/ha at } 30 \text { DAP. }\end{array}$ & 65.24 & 58.72 \\
\hline $\mathbf{T}_{6}$ & $\begin{array}{l}\text { Alachlor 50\% EC @ } 2.5 \text { litre/ha + Quizalfop Ethyl } 5 \\
\text { EC@ 1000ml/ha at } 30 \text { DAP. }\end{array}$ & 60.36 & 41.35 \\
\hline $\mathbf{T}_{7}$ & $\begin{array}{l}\text { Butachlor 50\% EC @ } 2.5 \text { litre/ha + Quizalfop Ethyl } 5 \text { EC@ } \\
\text { 1000ml/ha at } 30 \text { DAP. }\end{array}$ & 47.76 & 44.68 \\
\hline $\mathbf{T}_{8}$ & $\begin{array}{l}\text { Diuron 80\%WP @ 1.25 kg/ha + Quizalfop Ethyl } 5 \text { EC@ } \\
\text { 1000ml/ha at } 30 \text { DAP. }\end{array}$ & 75.6 & 53.9 \\
\hline $\mathbf{T}_{9}$ & Pendimethalin 30\% EC @ 3litre/ha + HW at 30 DAP. & 78.65 & 78.72 \\
\hline $\mathbf{T}_{10}$ & Alachlor 50\% EC @ 2.5 litre/ha + HW at 30 DAP. & 71.95 & 55.32 \\
\hline $\mathbf{T}_{11}$ & Butachlor 50\% EC @ 2.5 litre/ha + HW at 30 DAP. & 72.35 & 69.15 \\
\hline $\mathbf{T}_{12}$ & Diuron 80\%WP @ 1.25 kg/ha + HW at 30 DAP. & 80.08 & 65.25 \\
\hline $\mathbf{T}_{13}$ & Once hand weeding at $30 \mathrm{DAP}$ & 76.22 & 58.58 \\
\hline $\mathbf{T}_{14}$ & Weedy check (control) & 0 & 0 \\
\hline
\end{tabular}


Table.6 Seedling height at different stages of growth as influenced by weed control treatments in secondary nursery of Red Sander and Teak

\begin{tabular}{|c|c|c|c|c|c|c|c|c|}
\hline \multirow{3}{*}{ Treatments } & \multicolumn{4}{|c|}{ Red Sander } & \multicolumn{4}{|c|}{ Teak } \\
\hline & \multicolumn{4}{|c|}{ Seedling's height (cm) } & \multicolumn{4}{|c|}{ Seedling's height (cm) } \\
\hline & Initial & 1 MAT & 2 MAT & $\%$ improvement & Initial & 1 MAT & 2 MAT & $\%$ improvement \\
\hline $\mathbf{T}_{1}$ & 14.59 & 15.59 & 16.55 & 13 & 21.88 & 23.06 & 24.8 & 13.34 \\
\hline $\mathbf{T}_{2}$ & 16.24 & 17.73 & 17.95 & 10.5 & 23.75 & 25.04 & 26.68 & 12.33 \\
\hline $\mathbf{T}_{3}$ & 14.4 & 14.89 & 15.36 & 7 & 26.25 & 27.38 & 28.43 & 8.3 \\
\hline $\mathbf{T}_{4}$ & 10.92 & 11.84 & 12.6 & 15 & 28.67 & 28.97 & 29.96 & 4.49 \\
\hline $\mathbf{T}_{5}$ & 13.25 & 14.16 & 15.03 & 13 & 25.33 & 26.5 & 27.93 & 10.26 \\
\hline$T_{6}$ & 15.17 & 16.02 & 17.45 & 15.03 & 27.92 & 28.85 & 29.61 & 6.05 \\
\hline $\mathbf{T}_{7}$ & 13.59 & 14.92 & 15.32 & 13 & 20.33 & 20.96 & 22.92 & 12.73 \\
\hline $\mathbf{T}_{8}$ & 16 & 17.25 & 17.95 & 12 & 27.58 & 27.82 & 29.87 & 8.3 \\
\hline $\mathbf{T}_{9}$ & 14.09 & 15.17 & 16.19 & 14 & 21.75 & 24.91 & 26.33 & 21.05 \\
\hline $\mathbf{T}_{10}$ & 13.92 & 14.46 & 15.65 & 12 & 19 & 19.53 & 20.94 & 10.21 \\
\hline$T_{11}$ & 10.25 & 10.83 & 11.71 & 14 & 21.75 & 22.18 & 24.02 & 10.43 \\
\hline $\mathbf{T}_{12}$ & 17.25 & 18.65 & 19.9 & 15.4 & 27 & 29.02 & 30.82 & 14.14 \\
\hline$T_{13}$ & 12 & 12.68 & 13.19 & 10 & 31.33 & 33.8 & 35.33 & 12.76 \\
\hline $\mathbf{T}_{14}$ & 10.58 & 11.21 & 11.29 & 6.7 & 26.5 & 27.2 & 27.44 & 3.54 \\
\hline SE $\mathbf{m} \pm$ & 1.32 & 1.362 & 1.398 & - & 3.383 & 3.616 & 3.655 & - \\
\hline $\begin{array}{l}\text { C.D. at } \\
5 \% \text { level }\end{array}$ & NS & 4.171 & 4.282 & - & NS & NS & NS & - \\
\hline
\end{tabular}

Table.7 Collar diameter at different stages of growth as influenced by weed control treatments in secondary nursery of Red Sander and Teak

\begin{tabular}{|c|c|c|c|c|c|c|c|c|}
\hline \multirow{3}{*}{ Treatments } & \multicolumn{4}{|c|}{ Red Sander } & \multicolumn{4}{|c|}{ Teak } \\
\hline & \multicolumn{4}{|c|}{ Collar diameter $(\mathrm{cm})$} & \multicolumn{4}{|c|}{ Collar diameter $(\mathrm{cm})$} \\
\hline & Initial & 1 MAT & 2 MAT & \% improvement & Initial & 1 MAT & 2 MAT & \% improvement \\
\hline $\mathbf{T}_{1}$ & 0.25 & 0.27 & 0.29 & 16 & 0.51 & 0.549 & 0.585 & 14.7 \\
\hline $\mathbf{T}_{2}$ & 0.45 & 0.508 & 0.543 & 20.67 & 0.405 & 0.424 & 0.443 & 9.38 \\
\hline $\mathbf{T}_{3}$ & 0.315 & 0.354 & 0.367 & 16.5 & 0.62 & 0.646 & 0.715 & 15.32 \\
\hline $\mathbf{T}_{4}$ & 0.35 & 0.367 & 0.409 & 16.8 & 0.615 & 0.643 & 0.678 & 10.24 \\
\hline $\mathbf{T}_{5}$ & 0.455 & 0.494 & 0.52 & 16.9 & 0.47 & 0.50 & 0.565 & 20.21 \\
\hline $\mathbf{T}_{6}$ & 0.37 & 0.467 & 0.489 & 32.16 & 0.425 & 0.455 & 0.477 & 12.24 \\
\hline $\mathbf{T}_{7}$ & 0.265 & 0.282 & 0.314 & 18.5 & 0.365 & 0.382 & 0.392 & 7.39 \\
\hline $\mathbf{T}_{8}$ & 0.375 & 0.441 & 0.469 & 25.07 & 0.35 & 0.359 & 0.38 & 8.57 \\
\hline $\mathbf{T}_{9}$ & 0.32 & 0.328 & 0.418 & 30.6 & 0.5 & 0.566 & 0.627 & 25.4 \\
\hline$T_{10}$ & 0.415 & 0.485 & 0.528 & 27.23 & 0.455 & 0.482 & 0.52 & 14.28 \\
\hline$T_{11}$ & 0.21 & 0.245 & 0.261 & 24.29 & 0.52 & 0.532 & 0.598 & 15 \\
\hline$T_{12}$ & 0.45 & 0.525 & 0.598 & 32.9 & 0.55 & 0.594 & 0.643 & 16.9 \\
\hline$T_{13}$ & 0.275 & 0.325 & 0.341 & 24 & 0.37 & 0.436 & 0.441 & 19.19 \\
\hline $\mathrm{T}_{14}$ & 0.4 & 0.43 & 0.43 & 7.5 & 0.54 & 0.556 & 0.563 & 5 \\
\hline S.E.m \pm & 0.027 & 0.029 & 0.036 & - & 0.022 & 0.022 & 0.023 & - \\
\hline $\begin{array}{l}\text { C.D. at } \\
5 \% \text { level }\end{array}$ & 0.081 & 0.089 & 0.11 & - & 0.069 & 0.069 & 0.07 & - \\
\hline
\end{tabular}


The higher collar diameter and increment attained by red sander and teak could be due to reduced competition from less number of weeds for nutrients, space and light.

In this study, observation on weed flora indicated that 17 weed species belonging to 12 families were present in secondary nursery. Application of Pendimethalin with one hand weeding at 30 DAT showed maximum weed control efficiency in Secondary nursery of Teak while Diuron with one hand weeding at 30 days after primary treatment showed aggressive weed control efficiency in secondary nursery of red sander. Hence, integrated application of Pendimethalin and Diuron with one hand weeding at 30 DAT can be suggested to adopt in secondary forest nurseries of teak and Red sander respectively to get healthy and sturdy seedlings.

We want to emphasize the importance of careful selection of herbicides together with knowledge on species-specific, seedling phase requirement. Pre-emergence herbicides can effectively reduce or even eliminate weed seed bank in tropical forest nursery and also negatively affect seedling growth used in restoration projects.

\section{References}

Andrei, E., 2013, Compendio de defensives agricolas: guia pratico de produtos fitossanitarios para uso Agricola $\left(9^{\text {th }}\right.$ edition). Organizacao Andrei, Sao Paulo, pp 1577-1602.

D'Antonio, C. and Meyerson, L. A., 2002, Exotic plants species as problems and solutions in ecological restoration: a synthesis. Restor. Ecol., 10(4): 703713.

Hakim, M. A., Juraimi, A. S., Ismail, M. R., Hanafi, M. M. and Selamat, A., 2010, Distribution of weed population in the coastal rice growing area of Kehah in Peninsular Malaysia. J. Agron.,
9: 9-16.

Jinks, R. L., Willoughby, I. and Baker, C., 2006, Direct seeding of ash and sycamore: the effects of sowing date, pre-emergent herbicides, cultivation and protection on seedling emergence and survival. For. Ecol. Manage., 237(1): 373-386.

Panse, V. G. and Sukhama, P. V., 1967, Statistical Methods of Agricultural workers. ICAR, New Delhi.

Pereira, S. R., Laura, V. A. and Souza, A. L. T., 2013, Establishment of Fabaceae tree species in a tropical pasture: influence of seed sizes and weeding methods. Restor. Ecol., 21(1): 6774.

Rodrigues, R. R., Gandolfi, S., Nave, A. G., Aronson, J., Barreto, T. E., Vidal, C. Y. and Brancalion, P. H., 2011, Large scale ecological restoration of high diversity tropical forest in South East Brazil. For. Ecol. Manage., 261(10): 1605-1613.

Willoughby, I., 1996, Weed control when establishing new woodlands by direct seedling. Forestry Commission information note 286. Forestry Commission, Edinburgh.

Willoughby, I., Clay, D. V. and Dixon, F. L., 2003, The effect of preemergent herbicides on germination and early growth of broadleaved species used for direct seedling. Forestry., $\quad 76$ : 8394.

Willoughby, I., Jinks, R. L., Kerr, G. and Gosling, P. G., 2004a, Factors affecting the success of direct seeding for lowland afforestation in the United Kingdom. Forestry., 77: 467-482.

Willoughby, I., Jinks, R. L. and Stokes, V., 2006, the tolerance of newly emerged broadleaved tree seedlings to the herbicides clopyralid, cycloxydim and metazachlor. Forestry., 79(5): 599-608. 


\section{How to cite this article:}

Shahbaz Noori and Ramesh Rathod 2019. Weed Management in Secondary Nursery of Tectona grandis and Pterocarpus santalinus. Int.J.Curr.Microbiol.App.Sci. 8(09): 1437-1446. doi: https://doi.org/10.20546/ijcmas.2019.809.165 